\title{
Age and Race Impact the Association Between BMI and CVD Mortality in Women
}

Jill E. Abell, PhD, MPH BRENT M. EgAN, MD Peter W.F. Wilson, MD Stuart Lipsitz, ScD ${ }^{\mathrm{a}, \mathrm{b}}$ ROBert F. WOOLSON, PhD Daniel T. Lackland, DrPH

\section{SYNOPSIS}

Objectives. In previous studies, we have shown that obesity is associated with increased cardiovascular disease (CVD) mortality in white women but not in black women. Earlier research suggests that body mass index (BMI) has a greater effect on CVD mortality in younger white females than older white females, whereas this relationship in black women is not as clear. This study examines the effect of age on the association of BMI to CVD in black and white women.

Methods. The Black Pooling Project includes data on 2,843 black women with 50,464 person-years of follow-up, and 12,739 white women with 214,606 person-years of follow-up. A Cox proportional hazards model was used to examine the association between BMI and CVD mortality for specific age/race groups. The younger group was $<60$ years of age and the older group was $>60$ years of age.

Results. In younger white women, the relative risk ( $95 \%$ confidence interval [Cl]) for CVD mortality was significant in obese women (BMI $\left.>30 \mathrm{~kg} / \mathrm{m}^{2}\right)$ vs. women of normal weight (BMI 18.5-24.9 kg/m²) (1.59 [Cl 1.20, 2.09]). Similarly, in older white women, the relative risk for CVD mortality in obese women vs. women of normal weight was significant (1.21 [Cl 1.04, 1.41]). There were no such associations for black women. Overweight (BMI $\left.25-29.9 \mathrm{~kg} / \mathrm{m}^{2}\right)$ was not associated with increased risk in black or white women.

Conclusion. These findings indicate that obesity is associated with a significantly greater risk of CVD mortality among white women, with the strongest association among white women $<60$ years of age. 
Although obesity represents a health risk for most populations, the risk levels may vary with race and age. ${ }^{1}$ Some studies have found that the risk of cardiovascular disease (CVD) mortality is associated with a high body mass index (BMI), ${ }^{2}$ that the risk associated with obesity is greater for white respondents than black respondents, ${ }^{3}$ and that there is an independent, direct, positive association with BMI even after controlling for variables in the causal pathway. ${ }^{4}$

Age has been found to impact the association of body mass and CVD mortality. Analysis of the American Cancer Society's Cancer Prevention Study data found that among men and women aged 30-74 years, a greater body weight was associated with an increased risk of death from CVD, but that the risk associated with excess weight was greater in younger people. ${ }^{5}$ Data suggest that the prevalence of obesity decreases after age $60,{ }^{6}$ and it has long been questioned whether the ideal weight for elderly people should be greater than for younger people. ${ }^{7}$ Obesity also has a profound effect on life expectancy, especially among younger adults. ${ }^{8}$ However, in black respondents, decreased life expectancy was observed at higher BMI levels than in white respondents, suggesting that high body mass is not as detrimental in the black population.

Overweight and obesity is a growing epidemic and major public health concern. According to estimates from 2003-2004, 62\% of U.S. women were overweight or obese $\left(\right.$ BMI $\left.>25 \mathrm{~kg} / \mathrm{m}^{2}\right), 33 \%$ were obese (BMI $>30 \mathrm{~kg} / \mathrm{m}^{2}$ ), and $7 \%$ were morbidly obese (BMI $\left.>40 \mathrm{~kg} / \mathrm{m}^{2}\right) .{ }^{9}$ The prevalence of overweight and obesity varied with age and race. Overweight and obesity was greater in middle-aged respondents ( $40-59$ years) than in younger (20-40 years) and older ( $>60$ years) women, and the prevalence was higher in black respondents than in white respondents in all age groups. With the continuing rise in overweight and obesity, particularly in younger adults and children, it has been suggested that this may be the first generation to have a shorter lifespan than their parents. ${ }^{10}$

In previous studies, we have found that obesity is associated with CVD mortality in white respondents but not in black respondents. ${ }^{11}$ This analysis examines the effect of age on the association of BMI and CVD mortality in black and white women.

\section{METHODS}

\section{Data source}

This study uses data from the Black Pooling Project, which includes subject-level data from four studies: Evans County Heart Study, Charleston Heart Study, National Health and Nutrition Examination Survey
(NHANES) I, and NHANES II. The Black Pooling Project was established to provide an adequate number of black subjects to assess CVD risks. Baseline data on 15,582 women $(2,843$ black women and 12,739 white women) were collected between 1960 and 1980. Followup on mortality status lasted 15-30 years (Charleston Heart Study, 1960-1990; Evans County Heart Study, 1960-1990; NHANES I, 1971-1993; and NHANES II, 1976-1991) and includes 265,070 person-years.

In each of the studies, blood pressure, cholesterol, height, weight, diabetes status, and smoking status were measured or collected in a uniform manner by trained personnel. ${ }^{12-14}$ Cause of death was determined from death certificates in each study ${ }^{13-17}$ except NHANES II, in which mortality status was ascertained solely by computerized matching to national databases and evaluation of the resulting matches. ${ }^{18}$ In addition to death certificates, all information gathered in the Evans County Heart Study pertaining to death was reviewed by a panel of three physicians and a cardiologist to classify the deaths. A neurologist also reviewed the information for any evidence of stroke. ${ }^{16}$

\section{ANALYSIS}

Sample size, GVD deaths, person-years of follow-up, prevalence of overweight (BMI 25.0-29.9 kg/m²), obesity (BMI $>30 \mathrm{~kg} / \mathrm{m}^{2}$ ), and other study characteristics were determined for each age/race group. Hazard ratios, which are similar to relative risks in the Cox proportional hazard model and will thus forth be defined as a relative risk (RR), were estimated for CVD mortality associated with overweight and obesity using normal weight (BMI 18.5-24.9 $\mathrm{kg} / \mathrm{m}^{2}$ ) as a referent group. Three models-one controlling for age, the second controlling for age and smoking, and the third controlling for age, hypertension, cholesterol, diabetes, and smoking status-were run separately within the age/race groups in each of the four cohorts. Data were pooled using subject-level meta-analysis to obtain an overall estimate for each age/race group. The covariates in each model were selected a priori.

Model 1 provides a crude age-adjusted measure of risk. Model 2 controls for age and smoking; smoking is a common covariate because of its association with weight. Model 3 adjusted for age, hypertension, total cholesterol, diabetes, and smoking status to test for associations between BMI and CVD mortality after controlling for known cardiovascular risk factors. Differences in risk were tested with a z-statistic.

Subject-level meta-analysis was used to calculate the overall estimate of the weight effect. Subject-level meta-analysis involves the reanalysis of subject-level 
data, providing a reliable means of addressing questions not satisfactorily resolved in individual studies. ${ }^{19-24}$ This method is a more sensitive analysis than typical study-level meta-analysis. A model was fit for each study, age, and race group. The risk factor coefficients from the individual regression models were then combined to obtain a pooled coefficient. The pooled coefficients comprise the pooled risk equation for each age/race group. Both fixed and random effects models were used to obtain the pooled risk equation. Because the results from the fixed effect model were generally consistent with the results from the random effects model, only the results from the fixed effect model are reported.

\section{RESULTS}

The prevalence of obesity in black and white women in 10-year age groups is presented in the Figure. The prevalence of obesity in black women increased from $22 \%$ in those younger than 30 years to $42 \%$ in those aged 50-60 years. After age 60, the prevalence of obesity began to decline, and among those older than 70 years, 26\% were classified as obese. Among white women, obesity prevalence increased from $11 \%$ in those younger than 30 years to $21 \%$ in 50 - to 60-year-olds. After age 60 , the prevalence of obesity remained con- stant in white women rather than decreasing, as was seen in black women.

Table 1 presents sample size, CVD deaths, personyears of follow-up, mean, and prevalence of study characteristics for younger $(<60$ years $)$ and older $(>60$ years) black and white women. The prevalence of overweight, obesity, hypertension, and diabetes is greater in black women than in white women and increases with age. Current smoking was more prevalent in white women and decreased with age.

The relative risk of CVD mortality associated with BMI (overweight and obese) compared to normal weight was calculated for each age/race group in each of the four studies and pooled using meta-analysis (Table 2). There was little or no risk associated with overweight compared to normal weight in any of the four age/race groups. Among obese women, there was a significant association with CVD mortality in younger and older white women in Model 1, Model 2, and Model 3, and for younger black women in Model 1 and Model 2 but not in Model 3. The association (95\% confidence interval [CI]) between obesity and CVD mortality was significantly greater in younger white women than in older white women in Model 1 (2.12 [CI 1.64, 2.74] vs. 1.38 [CI 1.20, 1.59], $p=0.013$ ) and in Model 2 (2.49 [CI 1.91, 3.22] vs. 1.44 [CI 1.25, 1.65],

Figure. Obesity prevalence by age group in black and white women

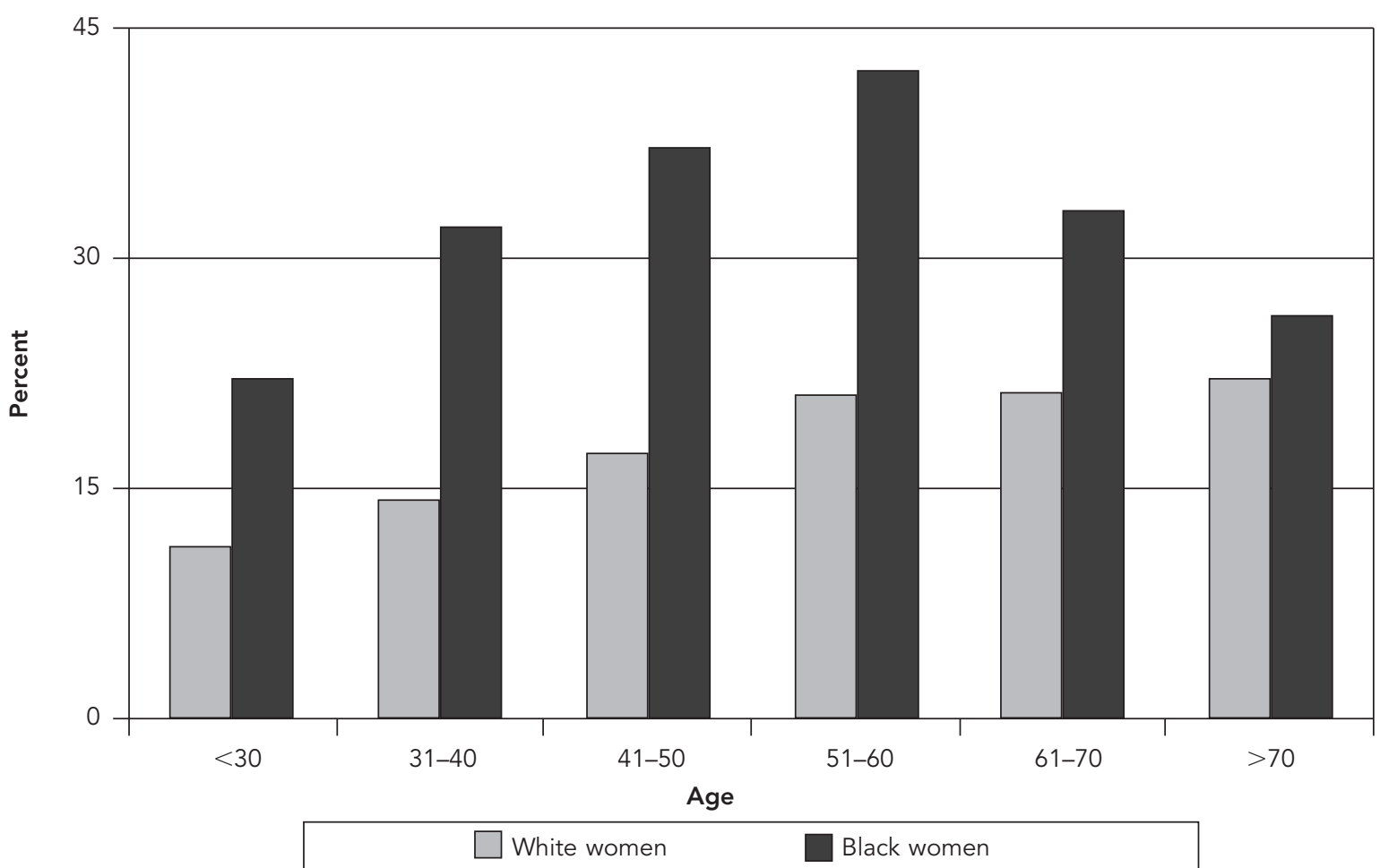

Public Health Reports / July-August 2007 / Volume 122 
Table 1. Study characteristics of younger and older black and white women

\begin{tabular}{|c|c|c|c|c|}
\hline & \multicolumn{2}{|c|}{ Age $<60$ years } & \multicolumn{2}{|c|}{ Age $>60$ years } \\
\hline & White & Black & White & Black \\
\hline$N$ & 8,189 & 1,953 & 4,490 & 864 \\
\hline CVD death & 378 & 266 & 1,259 & 360 \\
\hline Person-years & 152,922 & 38,969 & 61,684 & 11,496 \\
\hline Underweight (BMI <18.5 kg/m²) (percent) & 4.2 & 3.7 & 3.1 & 4.1 \\
\hline Normal (BMI 18.5-24.9 kg/m²) (percent) & 56.9 & 31.5 & 41.1 & 30.1 \\
\hline Overweight (BMI 25-29.9 kg/m²) (percent) & 23.2 & 30.1 & 34.3 & 34.1 \\
\hline Obese $\left(\mathrm{BMI}>30 \mathrm{~kg} / \mathrm{m}^{2}\right)$ (percent) & 15.7 & 34.7 & 21.5 & 31.7 \\
\hline Hypertensive (percent) & 26.7 & 54.4 & 62.5 & 80.4 \\
\hline Diabetes (percent) & 2.1 & 4.8 & 8.2 & 11.9 \\
\hline Current smoker (percent) & 34.7 & 34.3 & 16.3 & 12.3 \\
\hline Mean age (SD) & $41(10)$ & $42(9)$ & $67(4)$ & $67(4)$ \\
\hline Mean blood pressure (SD) & $125 / 80(20 / 12)$ & $142 / 90(31 / 16)$ & $148 / 85(25 / 12)$ & $165 / 92(32 / 16)$ \\
\hline Mean cholesterol (SD) & $215(48)$ & $214(47)$ & $249(49)$ & $243(49)$ \\
\hline
\end{tabular}

$\mathrm{BMI}=$ body mass index

$\mathrm{SD}=$ standard deviation

$p=0.002)$. The association between obesity and CVD mortality was greater for younger white women than for older white women in Model 3 (1.59 [CI 1.20, 2.11] vs. 1.21 [CI 1.04, 1.41], $p=0.126)$, but the difference was not significant. Obesity was associated with CVD mortality in younger but not older black women in Model 1 (1.39 [CI $1.03,1.90]$ vs. 0.99 [CI 0.77, 1.33]) and Model 2 $(1.46$ [CI $1.07,2.01]$ vs. 1.18 [CI $0.90,1.55])$. Obesity was not independently associated with CVD mortality in either younger or older black women in Model 3 (1.23 [CI $0.90,1.70$ ] vs. 0.99 [CI $0.75,1.33$ ]).

The association between obesity and CVD mortality was stronger in white women than in black women. This association was significantly greater in younger white women than in younger black women in Model 1 (2.12 [CI 1.64, 2.74] vs. 1.39 [CI 1.03, 1.90], $p=0.04$ ) and in Model 2 (2.49 [CI 1.91, 3.22] vs. 1.46 [CI 1.07, 2.01], $p=0.012)$, but not in the fully adjusted model, Model 3 (1.59 [CI 1.20, 2.11] vs. 1.23 [CI 0.90, 1.70], $p=0.238$ ).

\section{DISCUSSION}

In previous research, we found that obesity is associated with increased CVD mortality in white women but not in black women. ${ }^{11}$ This study extends the previous reports by documenting that obesity is associated with an increased risk of CVD mortality in both younger and older white women. Among white women, the association between obesity and CVD mortality is stronger in younger respondents than in older subjects. In black women, there was an association between CVD mortality and obesity in younger but not older women; however, this association was only significant in Model 1 (controlling for age) and Model 2 (controlling for age and smoking status). The association between obesity and CVD mortality was no longer significant in Model 3, which controlled for hypertension, total cholesterol, and diabetes in addition to age and smoking status. The results of Model 3 suggest that the increased CVD mortality risk in younger obese black women is mediated in part by risk factors associated with obesity, such as hypertension and diabetes.

These results suggest that the prevention and treatment of obesity would have the greatest impact on reducing total cardiovascular mortality in white women, especially those younger than 60 years. While there was a significant association between obesity and CVD mortality in both younger and older white women, the association was stronger in younger women. The observation that obesity is more strongly associated with CVD mortality in younger people is consistent with previous findings. For example, the Cancer Prevention Study found that elevated BMI was associated with increased CVD deaths but that the risk was greater in younger people. ${ }^{5}$

In a historical cohort of men with a mean follow-up of 35 years, a strong, significant association was found between BMI in young adulthood and CVD mortality (hazard ratio [HR] 2.41) and a weaker, insignificant association for BMI in mid-adulthood (HR 1.33). ${ }^{25} \mathrm{In}$ a prospective study of 6,193 obese patients, the risk of death increased with body weight, but obesity-related excess mortality declined with age at all levels of mortality. ${ }^{26}$ A report from the Longitudinal Study of Aging suggested that obesity might actually be protective in 
older community-dwelling Americans compared to being thin or of normal weight. ${ }^{27}$ The lack of association between CVD mortality and overweight in black and white respondents and obesity in black respondents is also consistent with previous findings. ${ }^{2,11}$ In the present study, this lack of association between overweight and CVD mortality persists among both younger and older subjects.

The suggestion that an increased body mass affects people differently is not a novel idea. There has long been the discussion that the ideal weight for elderly people should be greater than younger people, ${ }^{7}$ and it has been shown that the prevalence of obesity decreases after age $60 .{ }^{6}$ One explanation for this phenomenon is selective survival, in which obese young and middle-aged people die prematurely. A second possible explanation is a cohort effect, in which older people come from cohorts that typically were not obese, or body fat is redistributed with increasing age ${ }^{6}$ It is also possible that older subjects, even at a normal BMI, have less lean mass and more adiposity, which raises

\section{Table 2. Relative risk $(95 \% \mathrm{Cl})$ of CVD mortality associated with BMI in younger and older black and white women}

\begin{tabular}{lcc}
\hline Model 1a & $\begin{array}{c}\text { Overweight } \\
(95 \% \mathrm{Cl})\end{array}$ & $\begin{array}{c}\text { Obese } \\
(95 \% \mathrm{Cl})\end{array}$ \\
\hline White, age $<60$ years & $1.13(0.88,1.47)$ & $2.12(1.64,2.74)$ \\
Black, age $<60$ years & $1.09(0.78,1.53)$ & $1.39(1.03,1.90)$ \\
White, age $\geq 60$ years & $0.95(0.83,1.08)$ & $1.38(1.20,1.59)$ \\
White, age $\geq 60$ years & $0.82(0.63,1.07)$ & $0.99(0.77,1.33)$ \\
\hline
\end{tabular}

Model $2^{b}$

\begin{tabular}{lll} 
White, age $<60$ years & $1.28(0.99,1.67)$ & $2.49(1.91,3.22)$ \\
Black, age $<60$ years & $1.12(0.79,1.58)$ & $1.46(1.07,2.01)$ \\
White, age $\geq 60$ years & $0.98(0.85,1.12)$ & $1.44(1.25,1.65)$ \\
White, age $\geq 60$ years & $0.86(0.65,1.13)$ & $1.18(0.90,1.55)$ \\
\hline Model $3^{c}$ & & \\
\hline White, age $<60$ years & $0.99(0.76,1.29)$ & $1.59(1.20,2.11)$ \\
Black, age $<60$ years & $0.99(0.70,1.43)$ & $1.23(0.90,1.70)$ \\
White, age $\geq 60$ years & $0.91(0.79,1.05)$ & $1.21(1.04,1.41)$ \\
White, age $\geq 60$ years & $0.76(0.57,1.02)$ & $0.99(0.75,1.33)$ \\
\hline
\end{tabular}

${ }^{\text {a Adjusted for age }}$

${ }^{b}$ Adjusted for age and smoking status

cAdjusted for age, cholesterol, blood pressure, diabetes, and smoking status

NOTE: Underweight: BMI $<18.5 \mathrm{~kg} / \mathrm{m}^{2}$; normal weight: BMI 18.5$24.9 \mathrm{~kg} / \mathrm{m}^{2}$; overweight: BMI $25-29.9 \mathrm{~kg} / \mathrm{m}^{2}$; obese: $\mathrm{BMI}>30 \mathrm{~kg} / \mathrm{m}^{2}$ $\mathrm{Cl}=$ confidence interval

$C V D=$ cardiovascular disease

$\mathrm{BMI}=$ body mass index their CVD risk closer to levels of the obese. In this study, the prevalence of obesity peaked between 50 and 60 years of age in black women and then began to decline. However, in white women, the prevalence of obesity peaked at 50-60 years and then remained constant across the older age groups.

One of the limitations in this study was that all study characteristics were collected only at baseline. We did not have additional measures of the covariates to assess whether weight or other risk factor changes during the 15-30 years of follow-up might have affected the results. However, it has been suggested in a study of men that BMI in early adulthood is positively related to CVD mortality later in life, and that an association between weight gain and later mortality was not found. ${ }^{25}$ Although that study focused on men and not women, the results suggested that weight early in life might be a better predictor of CVD mortality than multiple measures or measures later in life.

Another limitation to this study was the lack of data on waist circumference, a commonly used measure of visceral fat. Previous reports indicated that a more centralized or abdominal fat pattern is associated with increased CVD mortality. ${ }^{28}$ Data on waist circumference may have allowed a more comprehensive analysis of the association between BMI and CVD mortality. However, BMI has been found to be highly correlated with waist circumference $(\mathrm{r} \sim 0.8-0.9),{ }^{29}$ and evidence suggests that waist circumferences at any BMI are similar or less in black respondents than in white respondents. ${ }^{30,31}$

One strength of this study was the lack of limitations we placed on our data. Analyses of weight and mortality often employ different methodology and lack uniformity, which does not allow comparison of results among various studies. For example, many studies limit their population to nonsmokers or exclude people who may have died early in the follow-up. In our study, we assessed the association of overweight and obesity and cardiovascular mortality among current, former, and nonsmokers. We did not exclude deaths that occurred early in follow-up. We controlled for smoking and other comorbid conditions in the analysis. This strategy provided results from a large number of black and white, younger and older women who are representative of the population.

These results suggest that there is an association between obesity and CVD mortality in younger and older white women. Among white women, obesity is associated with a greater CVD mortality risk in women younger than 60 years of age. This association is particularly important as the prevalence of obesity in children and younger adults being classified as overweight and obese continues to rise. As the prevalence of obesity 
increases in younger women, it is reasonable to assume that the number of women at increased risk of CVD mortality will also grow. Hence, obesity treatment and prevention would most likely have the greatest impact on reducing CVD mortality in white women, particularly those younger than age 60 .

While obesity was not independently associated with CVD mortality in black respondents, a significant association in younger black women was observed until adjustment was made for obesity-associated risk factors, including hypertension and diabetes. Because medical management of risk factors can be suboptimal, weight loss, which improves multiple cardiovascular risk factors, is likely to reduce cardiovascular mortality in younger black women. Given the noncardiovascular health risks of obesity, which include degenerative joint disease and several cancers, the treatment and prevention of obesity in all women has important personal and public health implications.

\section{REFERENCES}

1. National Heart, Lung, and Blood Institute (US). Clinical guidelines on the identification, evaluation and treatment of overweight and obesity in adults. The evidence report. 98-4083. National Institutes of Health (US); 1998.

2. McGee DL. Diverse population collaboration. Body mass index and mortality: a meta-analysis based on person-level data from twenty-six observational studies. Ann Epidemiol 2005;15:87-97.

3. Calle EE, Thun MJ, Petrelli JM, Rodriguez C, Heath CW Jr. Bodymass index and mortality in a prospective cohort of U.S. adults. N Engl J Med 1999;341:1097-105.

4. Dyer AR, Stamler J, Greenland P. Associations of weight change and weight variability with cardiovascular and all-cause mortality in the Chicago Western Electric Company Study. Am J Epidemiol 2000;152:324-33.

5. Stevens J, Cai J, Pamuk ER, Williamson DF, Thun MJ, Wood JL. The effect of age on the association between body-mass index and mortality. N Engl J Med 1998;338:1-7.

6. Seidell JC, Visscher TL. Body weight and weight change and their health implications for the elderly. Eur J Clin Nutr 2000;54(Suppl 3):S33-9.

7. Andres R, Elahi D, Tobin JD, Muller DC, Brant L. Impact of age on weight goals. Ann Intern Med 1985;103(Pt 2):1030-3.

8. Fontaine KR, Redden DT, Wang C, Westfall AO, Allison DB. Years of life lost due to obesity. JAMA 2003;289:187-93.

9. Ogden CL, Carroll MD, Curtin LR, McDowell MA, Tabak CJ, Flegal KM. Prevalence of overweight and obesity in the United States, 1999-2004. JAMA 2006;295:1549-55.

10. Olshansky SJ, Passaro DJ, Hershow RC, Layden J, Carnes BA, Brody J, et al. A potential decline in life expectancy in the United States in the 21st century. [see comment]. N Engl J Med 2005; 352:1138-45.

11. Abell JE, Wilson PWF, Egan BM, Lackland DT, Lipsitz SR, Woolson RF. Cardiovascular disease risks associated with weight differ for black and white men and women. American Heart Association Scientific Sessions; 2005 Oct 25. Abstract.

12. Johnson JL, Heineman EF, Heiss G, Hames CG, Tyroler HA.
Cardiovascular disease risk factors and mortality among black and white women aged 40-64 years in Evans County, Georgia. Am J Epidemiol 1986;123:209-20.

13. Keil JE, Sutherland SE, Hames CG, Lackland DT, Gazes PC, Knapp RG, et al. Coronary disease mortality and risk factors in black and white men. Results from the combined Charleston, SC, and Evans County, Georgia, heart studies. Arch Intern Med 1995; 155:1521-7.

14. Centers for Disease Control and Prevention (US). National Center for Health Statistics. National Health and Nutrition Examination Survey Data, 2006. Hyattsville (MD): Department of Health and Human Services (US); 2006.

15. Keil JE, Sutherland SE, Knapp RG, Lackland DT, Gazes PC, Tyroler HA. Mortality rates and risk factors for coronary disease in black as compared with white men and women. N Engl J Med 1993;329:73-8.

16. Cornoni JC, Waller LE, Cassel JC, Tyroler HA, Hames CG. The incidence study-study design and methods. Arch Intern Med 1971;128:896-900.

17. CDC (US). National Center for Health Statistics. National Health and Nutrition Examination Survey I data. Hyattsville (MD): Department of Health and Human Services (US); 2006.

18. CDC (US). National Center for Health Statistics. National Health and Nutrition Examination Survey II data. Hyattsville (MD): Department of Health and Human Services (US); 2006.

19. D'Agostino RB, Weintraub M. Meta-analysis: a method for synthesizing research. Clin Pharmacol Ther 1995;58:605-16.

20. Clarke MJ, Stewart LA. Obtaining data from randomised controlled trials: how much do we need for reliable and informative metaanalyses? BMJ 1994;309:1007-10.

21. Olkin I. Statistical and theoretical considerations in meta-analysis. J Clin Epidemiol 1995;48:133-46. Review.

22. Stewart LA, Parmar MK. Meta-analysis of the literature or of individual patient data: is there a difference? Lancet 1993;341:418-22.

23. Higgins JP, Whitehead A, Turner RM, Omar RZ, Thompson SG. Meta-analysis of continuous outcome data from individual patients. Stat Med 2001;20:2219-41.

24. DerSimonian R, Laird N. Meta-analysis in clinical trials. Control Clin Trials 1986;7:177-88.

25. Jeffreys M, McCarron P, Gunnell D, McEwen J, Smith GD. Body mass index in early and mid-adulthood, and subsequent mortality: a historical cohort study. Int J Obes Relat Metab Disord 2003; 27:1391-7.

26. Bender R, Jockel KH, Trautner C, Spraul M, Berger M. Effect of age on excess mortality in obesity. JAMA 1999;281:1498-504.

27. Grabowski DC, Ellis JE. High body mass index does not predict mortality in older people: analysis of the Longitudinal Study of Aging. J Am Geriatr Soc 2001;49:968-79.

28. Klein S, Burke LE, Bray GA, Allison DB, Pi-Sunyer X, Hong Y, et al. Clinical implications of obesity with specific focus on cardiovascular disease: a statement for professionals from the American Heart Association Council on Nutrition, Physical Activity, and Metabolism: endorsed by the American College of Cardiology Foundation. Circulation 2004;110:2952-67.

29. Harris MM, Stevens J, Thomas N, Schreiner P, Folsom AR. Associations of fat distribution and obesity with hypertension in a bi-ethinic population: the ARIC study. Obes Res 2000;8:516-24.

30. Zhu S, Heymsfield SB, Toyoshima H, Wang Z, Pietrobelli A, Heshka S. Race-ethnicity-specific waist circumference cutoffs for identifying cardiovascular disease risk factors. Am J Clin Nutr 2005; 81:409-15.

31. Okosun IS, Tedders SH, Choi S, Dever GE. Abdominal adiposity values associated with established body mass indexes in white, black and Hispanic Americans. A study from the third National Health and Nutrition Examination Survey. Int J Obes Relat Metab Disord 2000;24:1279-85. 